\title{
Escala de Felicidad Subjetiva: Validación en Puerto Rico
}

\author{
Subjective Happiness Scale: Validation in Puerto Rico
}

\section{Juan Aníbal González-Rivera ${ }^{1 *}$}

1 Ponce Health Sciences University, Ponce, Puerto Rico. (D) https://orcid.org/0000-0003-0622-8308

* Correspondencia: jagonzalez@psm.edu.

Recibido: 15 junio 2021 | Aceptado: 29 julio 2021 | Publicado: 15 agosto 2021

WWW.REVISTACARIBENADEPSICOLOGIA.COM

\section{Citar como:}

González-Rivera, J. A. (2021). Subjective Happiness Scale: Validación en Puerto Rico. Revista Caribeña de Psico-

logía, 5, e5753. https://doi.org/10.37226/rcp.v5i1.5753

\section{RESUMEN}

El presente estudio analiza las propiedades psicométricas de la Escala de Felicidad Subjetiva en una muestra de residentes de Puerto Rico. Un total de 300 adultos participaron en este estudio de carácter psicométrico. Los resultados de los estudios mostraron propiedades psicométricas adecuadas en la escala. El índice de confiabilidad Alfa de Cronbach de la escala fue de .91. La varianza explicada en el análisis factorial exploratorio fue de un 66.63\%. Los resultados confirmaron que la escala posee una estructura unidimensional. Los cuatro ítems de la escala cumplieron con los criterios de discriminación. Estos resultados sugieren que la escala tiene el potencial para medir este constructo en Puerto Rico.

Palabras Claves: felicidad, felicidad subjetiva, confiabilidad, propiedades psicométricas, validación

\begin{abstract}
This study analyzes the psychometric properties of the Subjective Happiness Scale in a sample of residents of Puerto Rico. A total of 300 adults participated in this psychometric study. The results of the studies showed adequate psychometric properties in the scale. The reliability index of the scale was of .91. The variance in the exploratory factorial analysis was $66.63 \%$. The results confirmed that the scale has a one-dimensional structure. The four items complied with the criteria of discrimination. These results suggest that the scale has the potential to measure this construct in Puerto Rico.
\end{abstract}

Keywords: happiness, subjective happiness, reliability, psychometric properties, validation

\section{INTRODUCCIÓN}

En las últimas décadas, gracias a la psicología positiva, han proliferado las investigaciones sobre la felicidad y, con ellas, el desarrollo de medidas que examinan distintas perspectivas del bienestar personal (González-Rivera, 2018). Entre estas se encuentra la felicidad subjetiva; constructo considerado como un fenómeno universal o global que permite que las personas puedan autoevaluarse e indicar si son o no felices. Entre los instrumentos que evalúan la felicidad desde esta perspectiva, se destaca la Escala de Felicidad Subjetiva (Subjective Happiness Scale) de Lyubomirsky y Lepper (1999) por su capacidad de evaluar los componentes afectivos y cognitivos del constructo únicamente con cuatro ítems. 
La escala fue validada originalmente en adultos de Estados Unidos y Rusia (Lyubomirsky \& Lepper, 1999) pero, por su brevedad y fácil administración, se han replicado estudios psicométricos alrededor del mundo, por ejemplo, Japón (Shimai et al., 2004), China (Swami, 2008), Austria y Filipinas (Swami et al., 2009), Portugal (Mota-Sousa, 2017; Spagnoli et al., 2010), Líbano (Moghnie \& Kazarian, 2012), Turquía (DoÄan \& Totan, 2013), Argentina (Ortiz et al., 2013) y Chile (Vera-Villarroel et al., 2011). Todos estos estudios han aportado evidencias internacionales de validez y confiabilidad.

Cabe destacar que no se encontraron estudios psicométricos realizados en Puerto Rico ni en otros países del Caribe. Tomando esto en consideración, el presente trabajo tuvo como objetivo analizar las propiedades psicométricas de la Escala de Felicidad Subjetiva en una muestra de residentes de Puerto Rico para sugerir su uso a la comunidad científica del país. Con este fin, se examinó la validez de constructo, la capacidad de discriminación de los ítems y la confiabilidad del instrumento.

\section{MÉTODO}

\section{Diseño de Investigación}

Se utilizó un diseño no experimental, transversal, de tipo instrumental (Ato et al., 2013) que fue aprobado por el Comité para la Ética en la Investigación de la Universidad Carlos Albizu en San Juan, Puerto Rico. La recopilación de datos se llevó a cabo mediante el uso de cuestionarios en línea y promociones a través de las redes sociales (e.g., Facebook, Instagram, Twitter, Google+, WhatsApp). La encuesta en línea incluía el consentimiento informado, el cual ofrecía todos los detalles del estudio. Para garantizar la privacidad y confidencialidad de los participantes, los cuestionarios se completaron de forma anónima.

\section{Participantes}

Se reclutó una muestra no probabilística de 300 adultos residentes de Puerto Rico, seleccionados por disponibilidad. En la Tabla 1 se presentan los datos sociodemográficos de la muestra.

\section{Instrumentos}

Escala de Felicidad Subjetiva. Este instrumento fue desarrollado por Lyubormirsky y Lepper (1999);
Tabla 1

Información sociodemográfica de la muestra.

\begin{tabular}{lcc}
\hline & $n$ & $\%$ \\
\hline Sexo & & \\
$\quad$ Mujer & 249 & 83.0 \\
$\quad$ Hombre & 51 & 17.0 \\
Edad & & \\
$\quad 21-30$ & 75 & 25.0 \\
$31-40$ & 112 & 37.3 \\
$41-50$ & 58 & 19.3 \\
51-60 & 47 & 15.7 \\
$\quad 61$ o más & 8 & 2.7 \\
Preparación Académica & & \\
$\quad$ Escuela superior o menos & 14 & 4.7 \\
Grado asociado o técnico & 14 & 4.7 \\
Bachillerato & 156 & 52.0 \\
$\quad$ Maestría/Doctorado & 116 & 38.7 \\
Ingresos anuales & & \\
\$0-20,000 & 82 & 27.3 \\
$\quad$ \$20,000-40,000 & 100 & 33.3 \\
$\quad$ \$40,000-60,000 & 65 & 21.7 \\
\$60,000-80,000 & 28 & 9.3 \\
\$80,000 o 100,000 & 9 & 3.0 \\
\$100,000 o más & 16 & 5.4 \\
\hline
\end{tabular}

el mismo es una medida global de felicidad subjetiva desde el juicio de quien responde. Consta de 4 ítems con una escala de respuesta tipo Likert de siete puntos cuya corrección se hace mediante la sumatoria de los puntos obtenidos en cada ítem. El primer ítem dicta "En general, me considero" y las opciones de respuestas van desde Una persona no muy feliz (1) a Una persona muy feliz (7). El segundo ítem pide al evaluado que se compare con otros, "Comparado con la mayoría de la gente que me rodea, me considero", y sus opciones de respuesta van desde Menos feliz (1) a Más feliz (7). El tercer y cuarto ítem ofrecen una descripción de personas felices/infelices y solicita a los encuestados que indiquen en qué punto se identifican con la premisa en una escala de respuestas que va desde Nada en $a b$ soluto (1) a En gran medida (7).

\section{Análisis de Datos}

Los datos se examinaron en IBM SPSS (versión 27), donde se realizaron análisis de discriminación de ítems, análisis de confiabilidad y un análisis factorial exploratorio. Para el análisis factorial exploratorio se utilizó el método de extracción de mínimos cuadrados no ponderados con rotación oblicua. Como 
criterios de aceptación, consideramos aquellos ítems con una carga factorial mayor a .50 en un solo factor (Stevens, 2002). El índice de discriminación se calculó con la correlación ítem total (rbis), el cual debe ser mayor a .30 (Kline, 2005). La confiabilidad se calculó con el coeficiente alfa de Cronbach y su versión estandarizada. Field (2013) y Kline (2000) indican que índices mayores a .70 son aceptables.

\section{RESULTADOS}

El análisis factorial exploratorio demostró una estructura unidimensional que explicaba el $66.63 \%$ de la varianza de los datos originales. Las pruebas de Kaiser-Meyer-Olkin $(\mathrm{KMO}=.778)$ y la prueba de esfericidad Bartlett $\left(X^{2}(45)=506.738, p<.000\right)$ apoyaron la adecuacidad de los datos de muestreo para el análisis. La Tabla 2 presenta las cargas factoriales obtenidas por los 4 ítems, así como los índices de discriminación y la varianza explicada por cada ítem. El Alfa de Cronbach obtenido fue de .81 y .83 en su versión estandarizada.

\section{Tabla 2}

Cargas factoriales, índices de discriminación y varianza explicada.

\begin{tabular}{|c|c|c|c|}
\hline Items & $r_{b i s}$ & $R^{2}$ & $C F$ \\
\hline 1. En general, me considero & .71 & .52 & .72 \\
\hline $\begin{array}{l}\text { 2. Comparado con la mayoría de la gente } \\
\text { que me rodea, me considero }\end{array}$ & .82 & .73 & .84 \\
\hline $\begin{array}{l}\text { 3. Algunas personas suelen ser muy felices. } \\
\text { Disfrutan la vida a pesar de lo que ocurra, } \\
\text { afrontando la mayoría de las cosas. ¿En qué } \\
\text { medida te consideras una persona así? }\end{array}$ & .80 & .68 & .82 \\
\hline $\begin{array}{l}\text { 4. Algunas personas suelen ser muy poco fe- } \\
\text { lices. Aunque no están deprimidas, no pare- } \\
\text { cen tan felices como ellas quisieran. ¿En qué } \\
\text { medida te consideras una persona así? }\end{array}$ & .87 & .80 & .89 \\
\hline
\end{tabular}

Nota: $r_{b i s}=$ Índice de discriminación; $R^{2}$ = Varianza explicada; $C F=$ carga factorial.

\section{CONCLUSIÓN}

Las propiedades psicométricas obtenidas en una muestra de adultos de Puerto Rico demuestran que la Escala de Felicidad Subjetiva refleja indicadores adecuados de validez y confiabilidad. Se concluye que la escala es una herramienta útil de medición para futuras investigaciones en el campo de la psicología positiva y de la psicología de la salud en Puerto Rico y el Caribe que traten el tema de la felicidad. Además, su lenguaje sencillo y su brevedad, consolida este instrumento como una de las primeras opciones al momento de realizar estudios que pretendan medir la felicidad.

Financiamiento: La presente investigación no fue financiada por alguna entidad ni patrocinador.

Conflicto de Intereses: No existen conflictos de intereses de parte del autor de la investigación.

Aprobación de la Junta Institucional para la Protección de Seres Humanos en la Investigación: Comité para la Ética en la Investigación de la Universidad Carlos Albizu en San Juan, Puerto Rico.

Consentimiento o Asentimiento Informado: Todo participante completó un consentimiento informado.

Proceso de Revisión: Este estudio ha sido revisado por pares externos en modalidad de doble ciego.

\section{REFERENCIAS}

Ato, M., López, J. J., \& Benavente, A. (2013). Un sistema de clasificación de los diseños de investigación en psicología. Anales de Psicología, 29(3), 1038-1059.

DoÄan, T., \& Totan, T. (2013). Psychometric properties of Turkish version of the Subjective Happiness Scale. The Journal of Happiness and Well-Being, 1(1), 21-28.

Field, A. P. (2013). Discovering statistics using IBM SPSS Statistics (4ª ed.). SAGE.

González-Rivera, J. A. (2018). Propiedades psicométricas de la Escala de Florecimiento en Puerto Rico. Revista Evaluar, 18(3), 3043.

Kline, P. (2000). Handbook of psychological testing. Routledge.

Kline, T. J. (2005). Psychological testing: A practical approach to design and evaluation. Sage.

Lyubomirsky, S., \& Lepper, H. S. (1999). A measure of subjective happiness: Preliminary reliability and construct validation. Social Indicators Research, 46(2), 137-155. https://doi.org/10.1023/A:1006824100041

Moghnie, L., \& Kazarian, S. (2012). Subjective happiness of Lebanese college youth in Lebanon: Factorial structure and invariance of the Arabic Subjective Happiness Scale. Social Indicators Research, 109(2), 203-210. https://doi.org/10.1007/s11205011-9895-5

Mota-Sousa, L. M., Marques-Vieira, C. M. A., Severino, S. S. P., Pozo-Rosado, J. L., \& Guerreiro-José, H. M.. (2017). Validación de la "Subjective Happiness Scale" en personas con Enfermedad Renal Crónica. Enfermería Global, 16(47), 38-70. https://dx.doi.org/10.6018/eglobal.16.3.266571

Ortiz, M. V., Gancedo, K. M., \& Reyna, C. (2013). Propiedades Psicométricas de la escala de felicidad subjetiva en jóvenes y adultos de la ciudad de Córdoba - Argentina. Suma Psicológica, 20(1), 45-56. 
Shimai, S., Otake, K., Utsuki, N., Ikemi, A., \& Lyubomirsky, S. (2004). Development of a Japanese version of the subjective happiness scale (SHS), and examination of its validity and reliability. Japanese Journal of Public Health, 51, 1-9. https://pubmed.ncbi.nlm.nih.gov/15565993/

Spagnoli, P., Caetano, A., \& Silva, A. (2010). Psychometric properties of a Portuguese version of the Subjective Happiness Scale. Social Indicators Research, 105, 137-143. https://doi.org/10.1007/s11205-010-9769-2
Stevens, J. (2002). Applied multivariate statistics for the social sciences (4⿳亠口冋口. ed.). Lawrence Erlbaum.

Swami, V. (2008). Translation and validation of the Malay Subjective Happiness Scale. Social Indicators Research, 88(2), 347-353. https://doi.org/10.1007/s11205-007-9195-2

Vera-Villarroel, P., Celis-Atenas, K., \& Córdova-Rubio, N. (2011). Evaluación de la felicidad: Análisis psicométrico de la Escala de Felicidad Subjetiva en población chilena. Terapia Psicológica, 29(1), 127-133. http://dx.doi.org/10.4067/S071848082011000100013

Obra bajo licencia de Creative Commons Atribución 4.0 Internacional (CC BY 4.0). (C) 2021 Autores. 